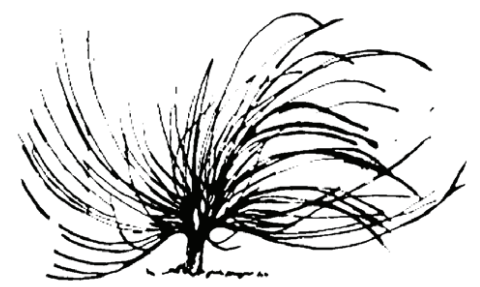

\title{
La Práctica Docente en el Área Secretarial: Un Ejercicio Decisivo para Minimizar la Tasa de Deserción Universitaria
}

\author{
Alba Canales García \\ Universidad Nacional \\ Heredia, Costa Rica \\ acanales25@gmail.com \\ Carolina España Chavarría \\ Universidad Nacional \\ Heredia, Costa Rica \\ cespanac@yahoo.com.mx
}

\begin{abstract}
Resumen
Estudio de caso realizado bajo la metodología de corte cualitativo y sobre lo vivenciado en el curso de práctica profesional. Se buscó conocer la percepción del grupo estudiantil respecto: a) la pertinencia de los aprendizajes promovidos y su correspondencia con las necesidades de aprendizaje del colectivo estudiantil como punto clave para minimizar la tasa de deserción, b) al valor otorgado al desarrollo del conocimiento y c) a la puesta en marcha de iniciativas de innovación que motiven al estudiante a
\end{abstract}

(c) (i) $\odot$

Recibido: 8 de octubre de 2014-Aprobado: 13 de abril de 2015

1 Máster en Educación con Énfasis en Docencia Universitaria, Licenciatura en Educación Comercial, experta en e-Learning; Docente e investigadora en la Universidad Nacional.

2 Doctora en Innovación Educativa. Docente e investigadora en la Universidad Nacional y la Universidad Estatal a Distancia de Costa Rica. Autora de múltiples artículos en periódicos, revistas y libros sobre el tema de educación. 
concluir sus estudios. Se concluye que los cursos de práctica profesional deben transformarse en espacios para la construcción del conocimiento del cual se derive un perfil profesional acorde con las demandas de significancia, autonomía y pertinencia que desafía la sociedad contemporánea. Todo esto como parte de las iniciativas de formación dirigidas a minimizar las tasas de deserción universitaria.

Palabras clave: práctica docente, aprendizaje autónomo, competencias, conocimiento, estudiante practicante, docente colaborador

\begin{abstract}
Case study conducted under the qualitative methodology and over the experience developed in the Professional Practice course. We wanted to study the perceptions of students regarding: the relevance of the promoted learning and its correspondence with the learning needs of the students as a key point to minimize the drop-out rate, the value given to the development of knowledge and the implementation of innovation initiatives that motivate students to conclude their university training. It is concluded that professional practice courses must become spaces for the construction of knowledge from which a profile is derived. This profile must meet the demands of significance, autonomy and relevance that contemporary society demands, all this as part of training initiatives aimed to minimized dropout rates in higher education.
\end{abstract}

Keywords: professional practice, autonomous learning, competencies and knowledge, intern student, teaching collaborator

\title{
1. Introducción
}

La deserción escolar ha sido un problema enfrentado por los colegiales en Costa Rica, según datos del Departamento Estadístico del Ministerio de Educación Pública (MEP) revelados por Guerrero en el sitio informativo crhoy.com el pasado 16 de julio del 2013. En este espacio se comunica que al finalizar las vacaciones de medio periodo del año 2013, la cifra de estudiantes que no regresaron al salón de clase fue de 
37.762. Sin embargo, la situación no era así de preocupante para quienes fueron parte del sistema de educación superior entre el 2000 y 2011. Esta información la confirma el último informe del Estado de la Educación en Costa Rica emitido en el año 2013. Los datos establecen que entre esos periodos, la población que asistía a la universidad incrementó en un 49,1\% y ascendió a 199.489 personas en el último año del período. También creció la asistencia a la educación parauniversitaria, pero en una proporción menor: $23,2 \%$. Ahora bien, el pasado 26 de mayo del 2014, según palabras de Isabel Román coordinadora de ese mismo informe emitidas para el periódico La Nación de Costa Rica, el asunto no debe enfocarse en la deserción como el fin último del problema sino más bien en la calidad educativa promovida como el objetivo por atender. Con esto será posible que el grupo discente termine sus estudios en el tiempo que se tiene establecido por las entidades educativas.

A pesar de que los datos suponen ser positivos para el sector universitario, es importante que los centros de formación no bajen la guardia en torno al tema de la deserción estudiantil. Por el contrario, se hace necesario el aseguramiento por parte de las universidades de que la población estudiantil matriculada en las diferentes carreras se mantenga activa a lo largo de todo el tiempo estimado para la conclusión de sus estudios. De esta manera, será posible controlar las tasas de deserción universitaria, las cuales aunque no son alarmantes al momento, se podrían disparar como consecuencia del alto costo de la vida, el cual obliga a muchos a dejar los estudios para iniciar su vida laboral.

Estas y otras consideraciones son parte de las preocupaciones de análisis y mejora que atiende la Universidad Nacional. Por tanto y en aras de atender a las demandas actuales para la formación universitaria del educador comercial, la investigación realizada en ese ámbito o contexto tuvo como objetivo principal analizar la opinión del estudiantado sobre el proceso de formación puesto en práctica en el curso Desafíos Didácticos y su correspondencia con los criterios de calidad educativa como punto clave para minimizar las tasas de deserción estudiantil. Para ello, se plantearon los siguientes objetivos específicos:

1. Conocer la percepción del alumnado respecto a la pertinencia de los aprendizajes promovidos en el curso Desafíos Didácticos en la Práctica Docente en Educación Comercial y su correspondencia 
con las necesidades de aprendizaje como punto clave para minimizar las tasas de deserción estudiantil.

2. Identificar el valor otorgado al desarrollo del conocimiento en la práctica pedagógica mediante las experiencias de aprendizaje del grupo de estudiantes del curso Desafíos Didácticos en la Práctica Docente en Educación Comercial.

3. Determinar la incidencia de los aprendizajes promovidos en el curso Desafíos Didácticos en la Práctica Docente en Educación Comercial para la puesta en marcha de iniciativas de innovación educativa en los contextos de formación.

\section{Marco teórico}

Abordar el tema de la deserción estudiantil va más allá de darle un tratamiento numérico a los datos que emite la matrícula inicial versus el número de graduados. Para esta investigación, lo urgente es pasar de lo cuantitativo a lo cualitativo, con el fin de poder entender cuáles son algunas de las razones que impulsan al grupo estudiantil a suspender sus estudios.

La interrupción del plan de estudios no debe ser atribuida a una sola causa, pues tanto factores internos como externos intervienen en esta realidad. Para muchos estudiosos en la materia, existe un fuerte ligamen de este hecho con los enfoques de enseñanza y aprendizaje utilizados en la educación superior, especialmente, si los analizamos a la luz de los nuevos paradigmas educativos, los cuales se orientan a mejorar las intervenciones didáctico-pedagógicas promovidas.

Sobre este tema, existen algunos casos de innovaciones curriculares y metodológicas dirigidas a incrementar la calidad de los cursos de investigación ofertados para el desarrollo de los trabajos finales de graduación (TFG) y con ello contribuir a que los requisitos para la conclusión de la licenciatura, como lo es el TFG, se realice de forma efectiva y placentera para el grupo estudiantil. Uno de estos es el caso de la Escuela de Secretariado Profesional de la Facultad de Ciencias Sociales en conjunto con la División de Educología del CIDE (Centro de Investigación y Docencia). Estos centros de estudio no solo comparten la carrera de Educación Comercial (Educología es responsable del componente pedagógico y Secretariado del área técnica), sino que ambos también han realizado un análisis de la situación actual enfrentada por los estudiantes en su último año de carrera, momento en el cual deben realizar su TFG 
para poder graduarse. Recientemente, según consta en el acta número 07 correspondiente a la Asamblea Ordinaria de Unidad Académica de la Escuela de Secretariado celebrada el pasado miércoles 15 de octubre del año 2014, en cumplimiento de lo que establece el artículo 113 del Estatuto Orgánico de la Universidad Nacional, se presentaron las cifras correspondientes a la matrícula actual y la proyección para el 2015. Estos datos se complementaron con las participaciones estudiantiles en relación con su frustración por no poder concluir sus estudios en el tiempo que inicialmente se les proyectó por el hecho de no contar con la formación y la guía idónea para desarrollar el TFG. Dicha situación provocó que los centros de estudio diseñaran una propuesta de intervención didáctico-metodológica para ser implementada a partir del 2015 en los seminarios de investigación. El objetivo consiste en que los estudiantes puedan iniciar con el trabajo de graduación una vez concluido el nivel de bachillerato bajo una metodología de integración teórico-disciplinar ajustable a sus intereses y necesidades técnicas y pedagógicas.

Cabe mencionar que para esta etapa se valoró también la calidad de la formación desplegada en el curso de práctica docente impartido en el nivel de bachillerato de la carrera de Educación Comercial, ya que las experiencias de campo que se desarrollaron en su momento como consecuencia de la mediación pedagógica realizada por el grupo formador le permite al estudiante en nivel de licenciatura, utilizar lo aprendido como parte de su madurez laboral y personal, sirviendo como elemento motivador para el grupo meta. Sin duda alguna, la formación que genera los aprendizajes construidos a partir de la dualidad entre el contexto laboral y el recinto universitario le conduce al estudiante aprender activamente mientras implementa el plan didáctico, las TIC (Tecnologías de la Información y Comunicación), los contenidos teórico-disciplinares, entre otros. Lo anterior contribuye a desarrollar una mejor autoestima y liderazgo, lo que a su vez, contribuye a minimizar las tasas de deserción universitaria.

Otro ejemplo importante de destacar es el estudio realizado por Canales y Ugalde en el año 2007. Las autoras titularon su obra Algunas razones asociadas a la deserción universitaria en el nivel de licenciatura: El caso de la Escuela de Bibliotecología, Documentación e Información. Bibliotecas. Su interés fue detectar la deserción de los estudiantes, posteriormente, tomar las medidas necesarias para evitar los niveles de deserción que se estaban presentando en ese momento. 
Ahora bien, las intenciones que persigue la investigación que se presenta en este artículo, conducen a evidenciar la pertinencia de los aprendizajes desplegados en el curso Desafíos Didácticos en la Práctica Docente, en Educación Comercial. Este es uno más de los requisitos que contiene el pensum académico de la carrera de Educación Comercial, de la Universidad Nacional de Costa Rica, a nivel de Bachillerato. El estudiante debe matricular esta asignatura en el I ciclo de su formación, y su importancia como parte de las iniciativas de formación, va dirigida a minimizar las tasas de deserción universitaria.

El curso en estudio se orienta a servir de espacio para la elaboración del pensamiento práctico en el estudiante, en donde los aprendizajes y el abordaje metodológico desplegado por el tutor(a) suponen estar dirigidos al desarrollo de un modelo de enseñanza y aprendizaje poco simple y unívoco, pues no solo está destinado a la inducción de los futuros educadores en el contexto educativo (en cualquiera que sea el nivel para el cual se forma: preescolar, primaria, secundaria o universidad), sino que supone enfocarse a la promoción de un trabajo más cooperativo, en donde el discente como futuro profesional de la educación resulte ser capaz de desarrollar estrategias que le permitan mejores prácticas.

El valor otorgado al aprendizaje y sus procesos de construcción será el resultado de experiencias relevantes y contextualizadas, que se desarrollen en el contexto escolar, el cual es concebido como el laboratorio de aprendizaje para el colectivo estudiantil. Lo vivido en esta fase formativa será un condicionante clave para elevar el sentido de pertinencia con las funciones profesionales que desarrollará en su vida futura, empoderar al estudiante de una mayor autonomía de pensamiento y acción, así como también, permitir un relacionamiento continuo de teoría y práctica. Todas estas bondades del proceso servirán para contrarrestar algunas de las causas que motivan la deserción educativa.

El curso analizado para este estudio, es el espacio que sirvió para la construcción autónoma del conocimiento. Sin duda alguna, este es un espacio para la socialización docente en el que se puede aprender a trabajar y concebir la actividad educativa de forma innovadora, sin imitaciones o reproducciones. Se busca además potenciar lo aprendido lejos de modelos de formación carentes de sustento y correspondencia con las demandas de significancia y pertinencia que reta la era contemporánea.

La situación anterior ha sido abordada por múltiples autores (Sepúlveda, 2005; Pérez, 1997; Caporossi, 2011 y Martínez, 2011), 
quienes valoran la urgente necesidad de conducir los procesos de formación, promovidos en el curso en estudio, desde el apoyo conceptual y teórico de la investigación como un acompañante sistemático y riguroso del proceso de socialización; los autores advierten que podría no solo existir un abordaje profundo y consensuado de los aprendizajes, sino también podría servir para que el docente novel minimice la instintiva tendencia (producto de modelos de formación tradicionales, que considera la transmisibilidad del conocimiento como condicionante, a su vez, la convierte en la forma para desarrollar la docencia) a la posible reproducción de vicios, prejuicios y mitos de orden procedimental, actitudinal y epistemológico.

Uno de los objetivos primordiales de acercar la formación universitaria al aula del centro escolar, es procurar servir de laboratorio de aprendizajes para el estudiante. Esta dualidad entre la universidad y la escuela no solo permite relacionar las experiencias de vida (modelo de formación, de socialización y demás) como sujeto en formación durante prácticamente toda su vida (preescolar, primaria, secundaria y universidad), sino también facilita el poner en práctica los conocimientos teóricos específicos que ha adquirido durante los cuatro años de carrera ${ }^{3}$, como eje medular para combatir cualquier intención de deserción estudiantil.

Por lo tanto, se considera que el curso Desafíos Didácticos debe aprovecharse como el espacio lúdico por excelencia, en el que los estudiantes pueden construir y reconstruir de forma integral y libre su propio perfil profesional. Para ello, es necesario que durante el periodo formativo, el estudiantado comprenda lo que acontece en su realidad profesional y personal. Lo anterior como resultado de su inmersión en el contexto educativo en donde se promueve su práctica docente. Este espacio sirve de escenario para conocer las responsabilidades que deberá asumir como docente, todas ellas relacionadas con el planeamiento, la atención de asuntos disciplinarios, el desarrollo de actividades lúdicas, la conducción de actos cívicos, la atención a padres de familia, la resolución de conflictos en condiciones habituales o por una emergencia, la conducción de reuniones con padres de familia, la asistencia y coordinación entre colegas y otras actividades. Las tareas profesionales aquí mencionadas se relacionan con un deber hacer docente propio de

3 El Bachillerato en Educación Comercial tiene una extensión de cuatro años, siempre y cuando curse la totalidad de asignaturas que establece cada bloque semestral que establece el programa. 
un contexto y un tiempo en particular por lo que difícilmente se encontrarán en libros de texto.

Tener la posibilidad de implementar nuevos enfoques de enseñanza para la promoción de los aprendizajes en los cursos de práctica docente, podría generar nuevas formas de socialización pedagógica en las que el estudiantado se enfrente a lecturas renovadas que le permitirían entender y aprender sobre su profesión de manera continua, dinámica y profunda, pues no solo atenderían al curso inmediato en el que se encuentra, sino que se orientarían a la construcción de competencias cognitivas y metacognitivas que le acompañarían toda su vida profesional, pues como profesional de una actividad meramente social, el futuro docente siempre estará en la necesidad de movilizar y adaptar sus conocimientos a las nuevas y complejas posibilidades, limitaciones, técnicas, actitudes y comportamientos de su ser profesional y de sus otros colegas, padres, directivos y estudiantes que como seres en evolución no solo le acompañarán, sino convertirán su función en un escenario multidinámico e inesperado. Por tanto, en la medida en que sepamos romper con las tradicionales formas de educar, estaremos evidenciado el sentido de aprender, este a su vez, será la razón primordial que mantendrá al grupo estudiantil activo en nuestros aulas universitarias hasta finalizar sus estudios.

\section{Marco metodológico}

El enfoque investigativo se realizó desde el paradigma naturalista. Hubo dominio del enfoque cualitativo y su realización se hizo bajo la metodología de estudio de caso de carácter descriptivo.

Cabe destacar que la investigación llevada a cabo, buscó interpretar la percepción del estudiantado, respecto al contraste experiencial entre lo vivido en un curso universitario y lo experimentado en la práctica docente que el curso les demanda asumir. Por lo que en ningún momento, se pretendió generalizar los resultados. Los hallazgos corresponden a las particularidades del caso estudiado; no obstante, es nuevo conocimiento que puede ser relacionado con situaciones similares y puede ser insumo para estudios de mayor profundidad. 


\subsection{Categorías de análisis}

Derivados de los objetivos que sirvieron de guía para este estudio, se distinguieron una serie de categorías de análisis ligadas al fenómeno de la deserción estudiantil, las cuales se citan a continuación:

a. proceso de formación desplegado

b. los criterios de calidad educativa

c. pertinencia de los aprendizajes promovidos

d. las necesidades de aprendizaje del colectivo estudiantil

e. valor otorgado al desarrollo del conocimiento en la práctica pedagógica

f. iniciativas de innovación educativa en los contextos de formación

\subsection{Participantes}

Los participantes son estudiantes de la carrera de Educación Comercial, del curso Desafíos Didácticos en la Práctica Docente en Educación Comercial, del nivel de bachillerato, ofertado entre la División de Educología y la Escuela de Secretariado Profesional de la Facultad de Ciencias Sociales, de la Universidad Nacional: 19 mujeres y un hombre pertenecientes al IV nivel de la carrera.

\subsection{Técnicas e instrumentos empleados para la recolección de datos}

El estudio de los objetivos planteados partió de la recolección de información mediante las técnicas de grupo focal en donde los estudiantes fueron seleccionados al azar y del cuestionario, este último instrumento se validó con docentes del área pedagógica y técnica.

La reflexión y la crítica fueron elementos claves para obtener y sistematizar la información empírica, junto con la aplicación de los instrumentos antes mencionados. Una vez concluida la jornada de clase regular; no hubo ningún estudiante que se abstuviera de formar parte de esta iniciativa de investigación, condición que facilitó el proceso de indagación y análisis.

El análisis de documentos permitió el contraste y la triangulación de los datos obtenidos de otras experiencias investigativas similares, de estudios y referencias teóricas sobre los principios básicos que caracterizan los enfoques de enseñanza y aprendizaje, apoyados en los nuevos paradigmas 
educativos. Paralelamente, se indagó en literatura diversa relacionada con el objeto del estudio, la calidad de la formación universitaria.

\section{Análisis de la información}

A continuación se presenta el análisis de la información recopilada con las técnicas aplicadas con el fin de responder a los objetivos planteados. Los datos emanados sirvieron de insumo para el desarrollo de las consideraciones finales presentadas en la etapa final de este documento.

La práctica docente es un ejercicio importante para el crecimiento profesional de los futuros docentes en el área de la Educación Comercial, a nivel de bachillerato, quienes, en esa etapa, ya han adquirido un bagaje de conocimientos, destrezas, valores y habilidades cognitivas y metacognitivas que les permiten no solo relacionar teoría y práctica, sino adentrarse de manera activa en el contexto de la función docente y así tomar decisiones relevantes orientadas a concluir de manera satisfactoria sus estudios. Lo valioso por destacar en este nivel de formación profesional, es la puesta en marcha de prácticas de trabajo profesional previamente planificadas por el instructor(a) del curso con el fin de posibilitarle al colectivo estudiantil los espacios requeridos para que de forma activa y vivencial amplíe sus conocimientos pedagógicos y los transfiera a un plano de mucho mayor significancia y funcionalidad para su vida profesional futura.

Asimismo, en esta etapa de formación, además de lo aprendido en la clase y fuera de ella, es de importancia el papel que juega el docente colaborador para la consecución de los objetivos que orientan el curso de práctica. Por consiguiente, este profesional es el que posibilita los espacios al estudiante para que de forma autónoma encuentre el sentido de aprender en un entorno universitario. Su acompañamiento le permitirá al grupo estudiantil desarrollar con éxito aprendizajes funcionales y significativos. Además, servirá para ejercer su futura función docente así como contrarrestar cualquier intento de abono formativo.

Lo anterior, explica la pertinencia del trabajo de campo realizado para esta investigación, pues se constituyó en medio que posibilitó contrastar lo que en el contexto de aula de curso de práctica sucede y lo que el estudiantado supone y aprecia como resultado de los aprendizajes promovidos y construidos en la clase del curso Desafíos didácticos en la práctica docente. 


\section{Presentación de la información}

A continuación, se presenta la información recabada por medio de las diferentes preguntas realizadas en el cuestionario (C), el grupo de discusión (GD), así como su relacionamiento con literatura sobre el tema meta.

\section{A.I. Descripción de la práctica docente}

El grupo de estudiantes encuestados describen la práctica docente como un espacio de aprendizaje, en donde la creatividad, el entusiasmo, la originalidad y la motivación son elementos que contribuyen a una promoción de aprendizajes para la vida mucho más funcional, significativa y pertinente. Otros por su parte consideran que es en este momento de su formación en donde se desarrolla el perfil actitudinal del docente (esta figura docente tiene la particularidad que cumple un doble papel, pues se difumina entre el estudiante y el profesional que será a futuro), es decir, sus competencias metacognitivas. Esto deja claro que para el estudiantado lo fundamental de describir la práctica no está limitado solo al contexto laboral, sino a las cualidades y capacidades del cuerpo docente como responsable activo de mediarla.

Entre las competencias y asuntos que destacan están las siguientes:

- La creatividad: al analizar cada manifestación de los estudiantes, se estima que un docente creativo es aquel que busca nuevas estrategias didácticas, recursos, empatía con los estudiantes, de tal forma que se logre promover un aprendizaje significativo.

- La paciencia: se denota en cuanto a la capacidad desarrollada para escuchar, a repetir los contenidos, instrucciones y el apoyo que brinda al estudiante en su crecimiento profesional.

- La autorrealización personal y profesional: el estudiante con esta virtud es aquel que se demuestra motivado con su práctica docente, por lo cual, puede rendir más en su tarea, sintiendo a su vez satisfacción por lo que hace. Se manifiesta dispuesto a provechar el apoyo y acompañamiento de los docentes (colaborador y tutor) para beneficio de su práctica.

- $\quad$ El sentido de responsabilidad se refiere a que el estudiante en el aula es visto con tal responsabilidad que, en algunos casos, se debe 
recurrir al distanciamiento, pues se incurre en acciones maternales, por tanto, el sentimiento de sobreprotección con los infantes, los invade haciendo que el objetivo de la práctica lo vean más como una responsabilidad que trasciende lo profesional y cae en preocupaciones derivadas de una relación madre-hijos o padre-hijos.

- La práctica docente: aunque es vista como parte fundamental en el proceso de enseñanza aprendizaje, es un espacio de aprendizaje necesario para aprobar el curso; asimismo, permite comprobar si realmente se ha aprendido durante los cuatro años de carrera, puesto que el aporte en aprendizaje que brinda es sustancial y transformador para su formación profesional y personal.

En segundo lugar, la práctica docente se ve como una enseñanza analítica, un reto y un proceso de aprendizaje. $\mathrm{Al}$ analizar lo que dichos atributos significan para el grupo meta como consecuencia del grupo de discusión sostenido, es posible darse cuenta nuevamente como las competencias del docente cobran protagonismo. Algunos ejemplos de lo comentado sobre cada aspecto:

- $\quad$ Enseñanza analítica: el estudiante practicante debe planificar cada lección con detenimiento, orden, a conciencia, buscando materiales, investigando sobre los contenidos, con disciplina, recursos tecnológicos y evaluación, entre otras consideraciones.

- Proceso de aprendizaje: todos aprenden de diferentes maneras, por lo que en el desarrollo de la práctica docente se continuarán aprendiendo, pues quienes practican se verán forzados a tomar decisiones, implementar innovaciones, ser críticos, analíticos e investigadores de su propia práctica, o bien, cuando un estudiante llega al aula y dice "profe, hoy me fue mal...; hoy me fue muy bien...; creo que la profesora colaboradora me calificó mal, creo que fallé en...; me calificó bien...", entre otros comentarios, eso permite que el espacio de aula sirva para la implementación de metodologías de aprendizaje colaborativo con el fin de formar al estudiante con una mentalidad cooperativa, de mayor apertura y socialización de sus prácticas de aprendizaje.

El mejoramiento de las prácticas docentes, modeladas desde el seno del aula formativa, podría contribuir a la construcción de un perfil 
profesional con visión más amplia del entorno educativo y de su realidad como ser humano y docente. El formar a un profesional para que aprenda a verbalizar su frustración, probablemente contribuirá a minimizar la tradicional cultura del aislamiento docente que por años se ha institucionalizado en los centros educativos; asimismo, quizás contribuya a la implementación de nuevos modelos de formación y asesoramiento de nuevas prácticas docentes como los ateneos didácticos propuestos por España (2012), en donde mediante la exposición e intercambio de ideas y experiencias profesionales los docentes mejoran su mediación pedagógica, pues en ellos hay apoyo y orientación mutua.

En tercer lugar, se encuentra el valor que se le da al estudiante en el entorno laboral de su práctica (en este caso podría ser en colegio técnico profesional, colegio vocacional, liceos en donde se imparte el área de tecnologías e institutos para-universitarios). Este aspecto es de gran importancia, debido a que el estudiantado de práctica se debe ver como parte medular del proceso de aprendizaje, en el que se le reconoce como futuro profesional de la docencia en su área (en una especialidad técnica, pues corresponde al área del secretariado profesional), por lo que el apoyo del profesor colaborador en la institución y el de quien ejerce como docente de práctica es imprescindible para su crecimiento y rendimiento en el espacio de práctica profesional.

Muchos estudiantes llegan al aula desmotivados porque no se les tomó en cuenta en el colegio donde están realizando su práctica. Un ejemplo de una estudiante es el siguiente: "En el primer día de clase, llegué temprano, preparé material, acomodé el aula y cuando llegaron todos los estudiantes, la profesora colaboradora me presentó como docente y no como estudiante de práctica, me sentí muy bien porque me integró al proceso" $(\mathrm{Gd} / \mathrm{e})$.

\section{B.I. Las dos cualidades que le distinguen como docente}

El criterio de la paciencia fue el de mayor nivel, aunque esté igual que los demás, está ligado a las competencias actitudinales que cuentan como sus cualidades distintivas. Su referencia se asocia al gusto por impartir clases, que a su vez, incluye respetar el ritmo de aprendizaje de los estudiantes, saber escucharlos y de ser posible aconsejarlos.

En un segundo nivel de prioridad, ubican la responsabilidad como un elemento importante, dado que para poder aprobar el curso de práctica 
docente deben cumplir con un tiempo, en este caso, impartir 50 lecciones sobre alguna subárea de Educación Comercial, en un colegio académico (diurno, nocturno) o instituto, todos estos de inversión pública. El cumplimiento con ir al día con los planeamientos de lección en cada clase, el organizar la clase con el acompañamiento del docente, el prepararse con anterioridad el material didáctico, el involucrarse en el diseño de los instrumentos de evaluación, así como otros asuntos, que son parte de lo que el docente colaborador valora y debe registrar como parte del desempeño del estudiante practicante. Esos serán los insumos sobre los cuales se discutirá y mediará en el curso universitario. Todo lo anterior, requiere un nivel de responsabilidad estudiantil muy elevado para que el proceso de práctica avance y pueda concluir satisfactoriamente.

En un tercer nivel, se sitúa la cualidad de ser cariñoso(a) con los estudiantes, pues es parte del proceso de enseñanza y aprendizaje y se deben tratar con cordialidad, amabilidad, con el fin de que se genere un ambiente agradable durante el desarrollo de la práctica.

Seguidamente, se ubica el ser estricto, este aspecto según lo externaron en el grupo de discusión (GD), se asocia a la necesidad de mantener un ambiente de respeto, de disciplina, en el cual el docente puede poner límites en el desarrollo de la clase; se concluyó que ser estricto, pero con amor es necesario, pues ambos criterios pueden convivir en el mismo nivel siempre.

El grupo meta aprovecha además del cuestionario y el grupo de discusión, la comunicación abierta con la profesora de curso (cumple doble función porque es también parte del equipo investigador) y le externa la importancia de que, además de lo discutido sobre el tema, como practicantes no deben de perder de vista la población estudiantil con la que se está trabajando en los centros educativos, y deben, además, tomar en cuenta que su propia condición de juventud podría facilitarles la interacción con el grupo de educandos. El grupo de estudiantes destacan la alta motivación que existe entre ellos, pues en muchos casos, este es el espacio idóneo para compartir sus intereses y realidades de vida pues sus argumentos son fácilmente comprendidos. También, debido a su gran afinidad por la tecnología y la innovación la población consultada considera muy positivo el hecho de que se les promueva una gran diversidad de recursos y actividades, las cuales les genera nuevas formas de construir aprendizaje.

Si bien es cierto, los ritmos y modos en que se construye el conocimiento son heterogéneos y obligan a generar acomodaciones en 
la práctica pedagógica, lo cual no impide que el profesional goce no solo de conciencia ante las necesidades de aprendizaje que existen, sino también que desarrolle una significativa entereza para su consecución.

En último orden de prioridad, se menciona que el docente debe ser práctico y divertido, lo cual podría ser consecuencia de una cualidad que se da por sentada en la actualidad que se aprecia de sus propios profesores de carrera, quienes, como consecuencia de procesos de formación y sensibilización docente, así como de un plan de estudios que maneja contenidos más prácticos que teóricos, por ser una especialidad de destreza, se han interesado más en que los alumnos aprendan en un ambiente cordial y ameno.

Para entender un poco más la explicación anterior, se aportará a manera de anécdota, un curioso recuerdo que brota en el triple papel de investigadora, estudiante de la carrera de Educación Comercial y docente del curso investigado. Se remonta a los años noventa, propiamente en la clase de destrezas mecanográficas, la cual podría ser descrita de la siguiente forma: la docente indicaba que las estudiantes debían copiar un texto en cierta cantidad de tiempo, ese tiempo lo medía con un reloj que emitía un fuerte sonido y si el estudiante continuaba digitando la profesora le demandaba con voz fuerte que se detuviera, generando en algunos casos una atmósfera de clase estresante. En la actualidad, esta situación no sucede, pues el uso de otros medios, actividades y actitudes por parte de los docentes es lo que prevalece, pues lo que se pretende es hacer la clase más significativa y atractiva para el grupo estudiantil.

Todas las cualidades anteriormente citadas por los estudiantes son valiosas en la actualidad, porque se genera una relación de aprendizajes significativos, necesarios para el mercado laboral, en donde el papel del docente es motivar, pues permite ser escuchado(a) y escuchar, acompañar y que lo acompañen en las tareas pedagógicas, así como generar en los y las futuros/as docentes el sentido de la responsabilidad para que sean mejores profesionales y personas.

\section{C.I. Promueve una formación para la vida}

De acuerdo con la información obtenida en el cuestionario respecto a la promoción de aprendizajes a tono con una formación para la vida, la mayoría de los estudiantes manifestaron que sí es posible alcanzar dicha meta, dado que en su práctica tratan de hacer las clases diferentes, en 
el sentido de motivar a educandos con actividades nuevas, manifestando interés por enseñar y que las clases no sean tan tradicionales (transmisoras de teorías enlatadas y preconcebidas) y se esfuerzan por crear un ambiente creativo para que el proceso de aprendizaje sea más fácil. Una de las manifestaciones que hizo un estudiante de la institución educativa fue que le gustaba que llegaran estudiantes de práctica a darles las clases porque llevaban cosas nuevas, como reflexiones, materiales, juegos; y en su mayoría, son jóvenes igual que ellos y eso los hacía sentir confianza e ir a la clase más motivados. Por otra parte, una minoría de los participantes consultados indicó que no se enseña para la vida, porque muchas veces se ven atados a cumplir con el programa académico y no da tiempo para lograr un ambiente más ameno para el aprendizaje.

En la actualidad, se necesita educar para la vida, proporcionar herramientas a los estudiantes que los faculten en la era tecnológica, motivarlos a descubrir, a innovar, a promover el sentido de la creatividad, que puedan enfrentar las necesidades educativas mediante la toma de decisiones, de tal forma, que sean los hombres y mujeres que la sociedad necesita, y poder así, ser parte de los docentes que requiere el siglo XXI.

\section{D.I. Lo que se necesita para realizar la práctica docente de manera satisfactoria}

Los estudiantes manifiestan en primera instancia que lo que necesitan es el respeto y la seguridad; ambos criterios nuevamente se relacionan con aspectos intangibles y curiosamente no están asociados al desarrollo de la intelectualidad. El grupo meta destaca la importancia de evidenciar que dicho respeto no solo se asocia a sus estudiantes, al docente colaborador(a) y al docente de curso; sino también al respeto que ellos se merecen en su lugar de práctica, pues alegan que a este punto de su transitar académico como universitarios(as), se sienten más profesionales que simples estudiantes observadores de una clase y, por tanto, se les debe considerar como tales. Lo anterior, se asocia a los niveles de seguridad manejados a la hora de impartir su clase, pues para muchos el respeto que se les dé depende de su desempeño práctico (cómo manejar los contenidos, qué tanto puedan aprender a aprender de acuerdo con las características de los grupos). Por tanto, respeto y seguridad son dos elementos que los practicantes ven como necesarios para que el proceso de su práctica sea más significativo e interesante. 
En segundo nivel de prioridad, el grupo meta ubica la humildad, la responsabilidad y la paciencia, como condicionantes para un desempeño exitoso de su práctica docente. El grupo entrevistado considera que sí el docente promueve la humildad dentro de su clase, sus estudiantes lo verán como alguien que sabe y que puede equivocarse, en lugar de que llegue como sí lo supiera todo con una actitud imponente poco conciliadora y elocuente. Con respecto a la responsabilidad, la consideran una cualidad necesaria dado que deben comprometerse con impartir las clases en el horario establecido, cumplir con exponer bien la materia, preparar su discurso, además de que deben dar cuentas al docente colaborador y a la profesora del curso sobre su avance y rendimiento en su trabajo de práctica.

Respecto a las evidencias encontradas en el trabajo de campo sobre las cualidades que distinguen al estudiante como docente, en un mayor porcentaje se menciona el ser responsable, condición que, como se puede apreciar, también está presente en la mayoría de las descripciones discentes ofrecidas. Se indicó que la responsabilidad es un elemento importante para realizar la práctica docente con éxito y lo ubican en un segundo lugar. Ahora bien, esta concepción de responsabilidad se hace mucho más importante cuando se le liga al componente ético de la función profesional, especialmente, para aquellos estudiantes que recién se inician como practicantes y deben coordinar con el director(a) de la institución, con el docente colaborador(a), organizar el material (planeamiento didáctico, copias, actividades, reflexiones etc.), además de cumplir con el curso universitario: Desafíos Didácticos. Así, uno de los informantes indicó: "Me propuse mejorar en el dominio de los temas, porque aunque uno conozca muy bien alguno en particular siempre es importante repasarlo y si hay palabras que uno no sabe buscarlas en el diccionario antes de darlo en una clase". (Gd)

El grupo participante del estudio ubica la preparación de material para impartir sus lecciones y el planeamiento respectivo en el último rango de prioridad, debido a que dichas tareas las dan por sentadas al conocerlas de previo. Cabe mencionar que parte de la formación recibida por el colectivo estudiantil en sus cuatro años de preparación universitaria, gira en torno a destacar las responsabilidades en la elaboración y diseño de los materiales que apoyan la práctica pedagógica, así como también a proveerle las herramientas necesarias para que desarrolle competencias actitudinales que le permitan entender la importancia 
de ser respetuoso, paciente, humilde y seguro de sus habilidades; de esta forma quizás, se constituya en un medio para que la enseñanza sea de provecho para todo el grupo de estudiantes que está aprendiendo.

\section{E.I. Fortalezas con las que cuenta para realizar la práctica docente}

Los resultados obtenidos en el trabajo de campo sobre este particular, demuestran que la mayoría de la población meta, se inclina entre las fortalezas con que cuenta para enfrentar los nuevos retos que les generará de su función como docentes. Las relaciones interpersonales, la dedicación, incentivar a los estudiantes para aprender juntos y el tono de voz a la hora de hablar son, además de otras, las cualidades que les serán de mucha utilidad, pues contribuyen a un mejor ejercicio de su función profesional en la educación.

Por otro lado, en una segunda fase la población participante del estudio opinó que la motivación es una fortaleza porque hará que sus clases sean amenas e interesantes. El docente motivado brindará un mejor trato al colectivo estudiantil y este podría, a su vez, llegar a sentirse parte del proceso de enseñanza y aprendizaje, con lo cual aumentaría su nivel de interés, contribuyendo a manera de efecto búmeran con el trabajo que realice el estudiante practicante, pues se desarrollaría en una ambiente más ameno y receptivo.

Seguidamente, una tercera parte de los encuestados manifestaron que el deseo de enseñar y aprender es una fortaleza con la que cuentan para enfrentarse a su práctica, pues es su primera experiencia en el campo de la educación formal y esa formación contribuirá a modelar una imagen de profesión y de profesional necesaria en su vida.

Por último, la muestra considera la preparación académica y el dominio de los temas como sus mayores fortalezas, razón que encuentra su fundamento en el tipo de formación que ofrece la Universidad, la cual se construye sobre un corpus teórico del cual se espera que de sus contenidos se deriven aprendizajes y de estos construcciones autónomas del conocimiento, adaptables y movilizables según las necesidades en los diferentes escenarios del contexto educativo. 


\section{F.I. Debilidades que el colectivo estudiantil dice tener para cumplir su labor con excelencia}

Por otra parte, y como una más de las consideraciones discentes están los contenidos y el tiempo como sus principales debilidades, seguido por la relación con los estudiantes y la autoridad. Su escogencia depende de algunos factores específicos tales como las características de los grupos y el manejo teórico-práctico, que tenga de los conocimientos en la disciplina meta, y aquellos requeridos para impartirla (las materias como matemática financiera, presupuesto y estadística, asignaturas ausentes en la malla curricular de la carrera de Educación Comercial, aunque son requeridas en los planes de estudio que el Ministerio de Educación Pública ofrece a los colegios técnicos. Esta incongruencia perjudica el desenvolvimiento profesional de los estudiantes practicantes.

Un caso concreto que ejemplifica lo comentado en el párrafo anterior, es el de una estudiante que debía impartir un determinado contenido teórico, pero el trasfondo cultural que complementaba la explicación del tema no era de su dominio, esto afectó la calidad de la exposición, y obligó a replantear su preparación para poder abordar el desarrollo temático de forma mucho más efectiva. Sin duda alguna, esta situación condujo a una inversión de tiempo mayor con el fin de poder buscar y seleccionar el material idóneo para cumplir con los requisitos de calidad que desafiaba su presentación. Lastimosamente, en este caso, el problema no se centró en la accesibilidad de fuentes informativas, sino más bien en lograr sintetizarlo para comprenderlo. Fallas como estas en conjunto con el manejo del estrés y la indecisión, incrementan la inseguridad, el descontento y la desmotivación del colectivo estudiantil provocando que este grupo muchas veces desista de continuar con sus estudios.

En un segundo lugar, la relación estudiante-docente es la mayor debilidad manifestada por el grupo participante del estudio. Para ellos, no existe empatía entre estudiantes y profesorado y eso incide a la hora de ir a impartir las lecciones. Para algunos, este aspecto requirió de mucha tolerancia a la hora de tomar el control del grupo, pues hubo casos como el de una estudiante de práctica, quien comentó en la clase su preocupación al respecto, pues sentía que no le caía bien a una estudiante y esto lo notaba como consecuencia de la conducta que la discente demostraba al interrumpirla cuando estaba desarrollando los contenidos 
con preguntas que no tenían relación con la materia, se burlaba de ella y generaba un ambiente de trabajo hostil.

Por otro lado, hubo quienes tuvieron experiencias contrarias e indicaron al final del curso, que sus vivencias fueron positivas gracias al apoyo de la docente colaboradora. Un caso en específico expuesto en el grupo de discusión revela lo siguiente: "Me siento muy bien, las profesoras me tratan de tú a tú, me aceptaron en el grupo como parte de una docente más. No me siento extraña, con gente extraña. Me siento en un grupo como si tuviera muchos años de estar con ellas. "(Gd)

Por último, los estudiantes meta indican que la falta de autoridad es una debilidad que debe ser valorada, pues puede influir a la hora de crear un ambiente de respeto. La autoridad entendida como liderazgo profesional podría generar modificaciones importantes en cómo se socializa en el contexto aprendiente, es decir, cuando el grupo estudiantil realiza sus actividades de clase. Al respecto, una estudiante relata en el grupo de discusión, que cuando le hicieron la evaluación final, las estudiantes la describieron como una profesional de carácter fuerte, pero a su vez, era cariñosa en el trato a los estudiantes por lo que recibir sus clases les era placentero.

\section{Consideraciones finales}

La práctica docente es un espacio importante para que el estudiante en formación pueda enfrentarse a la realidad de aula, es decir, comparta los conocimientos aprendidos durante su formación, relacionándose con estudiantes de secundaria y docentes en servicio, de tal manera, que se prepare, sea competente en un mercado laboral como lo es la docencia.

Para aquellos hombres y mujeres que solo son estudiantes universitarios y no han tenido la experiencia de laborar en el campo educativo, la práctica se constituye en su primer encuentro con la vivencia pedagógica. Por lo tanto, de esta experiencia deberían resultarle aprendizajes claves para fortalecer su formación profesional y personal, en forma crítica y constructiva que lo proyecten como el docente que se requiere en la actualidad. A su vez, debería convertirse en el espacio idóneo para asentar las responsabilidades del grupo estudiantil sobre su propia formación. En este nivel, los sujetos en formación tendrán más argumentos para decidir continuar o no con sus estudios, siendo entonces de gran trascendencia la calidad educativa desplegada. 
El compartir del practicante en la institución educativa con estudiantes y profesores del área, dará como resultado el reforzamiento de conocimientos y aprendizajes para la urgida transformación que requiere su perfil profesional.

Igualmente, dicha socialización en el contexto aprendiente podría contribuir a modelar su personalidad hacia estados de tolerancia, respeto, consideración y demás cualidades que como docente le serán valiosas para un mejor desempeño de su función pedagógica y en apego a los procesos de formación ofrecidos en el entorno universitario. En la medida en que el grupo estudiantil incorpore a su agenda personal y profesional la necesidad de transformarse, mediante estados de auto y mutua reflexión, sobre el qué y el cómo está llamado a hacer y ser en el seno pedagógico, su respuesta a las demandas que se le imponen como educador en la sociedad moderna serán quizás mucho más asertivas.

Bajo modelos de formación liberadores, significativos y funcionales será posible que el grupo discente desarrolle competencias claves para dar las soluciones idóneas a los problemas del entorno. También, le permitirá acceder con mucha más facilidad a nuevas formas en las que se informe, organice, diagnostique, diseñe materiales y ponga en marcha actividades, de las cuales le resulten ambientes pedagógicos más funcionales y significativos, contribuyendo a minimizar el riesgo potencial de deserción.

Por otra parte, la figura del docente colaborador se constituye en un elemento importante en el proceso, dado que es quien: a) orienta al estudiante para que pueda cumplir con los objetivos de formación trazados en el curso de práctica, y a la vez, b) apoya a que las lecciones sean más creativas, significativas, con actividades innovadoras, así como, c) evalúa el desempeño del practicante en el contexto real de aula mediante el instrumento que la docente, del curso Desafíos Didácticos diseña para tal fin, y con el cual, se determina parte de la calificación del estudiante.

En lo que se refiere al docente de curso, deberá en todo momento a) servir de mediador activo del proceso, b) orientar la práctica desde la experiencia académica, c) colaborar con escuchar las experiencias que sus estudiantes viven cada día en el contexto de práctica, sus alegrías, frustraciones entre otros y d) proponer soluciones a los conflictos que suelen emerger como resultado de la función práctica ejercida, como se reflejó en el grupo focal. 
El aprender a ser, a compartir, a convivir con otros (estudiantes, docentes, institución), a tener que tomar decisiones propias sobre su quehacer en las instituciones, a autoevaluarse en cuanto a su rendimiento como docente, a planear y organizar materiales, a distribuir el tiempo, a ver la realidad educativa con la que se cuenta en cada institución educativa son situaciones que hacen que se adquiera un distinto grado de madurez para enfrentar nuevos retos.

Además, es necesario que en el plan de estudios se proyecten materias que incluyan los contenidos que se imparten a nivel de secundaria, tales como presupuesto, contabilidad y matemática financiera, dado que al no contar con esos conocimientos, el estudiante practicante puede estar en desventaja teórica y práctica con otros estudiantes en su misma condición provenientes de universidades privadas, lo que resulte en frustración, estrés, ansiedad y falta de credibilidad en la formación universitaria recibida, además, de otros aspectos que podrían perjudicarle en su desempeño práctico, así como servir de justificante para dejar los estudios.

Por último, a lo largo de este proceso de indagación se evidencia la urgente necesidad de valorar el tipo de mediación y escogencia de las temáticas que los cursos de práctica profesional promueven, puesto que para este caso, en particular, fue de gran interés conocer el sentir del colectivo estudiantil, respecto a lo vivenciado en el curso "Desafíos Didácticos en la práctica docente para la Educación Comercial”, el cual fue orientado, además de lo que tradicionalmente se busca (mejorar la mediación pedagógica), al conocimiento teórico-práctico de los recursos y las herramientas tecnológicas con fines pedagógicos como aporte al proyecto de extensión que el estudiantado debe realizar (requerimiento del curso del práctica). Lo anterior hizo posible que el grupo estudiantil no solo cumpliera con el requisito de realizar su práctica en docencia en una institución educativa, sino también maximizara la utilización y aplicación de las TIC como apoyo a la práctica pedagógica, pues la mediación del curso les sirvió de modelo para conocer, aprender y poner en marcha, innovadoras prácticas de enseñanza y aprendizaje en sus contextos de práctica y así revelar el sentido funcional que tiene el aprender ininterrumpidamente a lo largo de todo su plan de estudios. 


\section{Referencias bibliográficas}

Canales, A y Ugalde, A. (2007). Algunas razones asociadas a la deserción universitaria en el nivel de licenciatura: El caso de la Escuela de Bibliotecología, Documentación e Información. Bibliotecas, $X X V(2)$, pp. 41-70.

Caporossi, A. (2011). La construcción del conocimiento profesional de la práctica docente in situ: construyendo conocimientos articulados y contextuados. Universidad Nacional de Rosario, Argentina; en Soubiron, E; Rodríguez, D; Sanz, V; Conde, A. (coords.) (2011). La práctica pedagógica en entornos innovadores de aprendizaje. Seminario Internacional. Montevideo, Uruguay.

Delors, J. (1994). Informe de la UNESCO. Recuperado de http://www. unesco.org/education/pdf/DELORS_S.PDF

Díaz, F. (2010). Modelo para autoevaluar la práctica docente. Gráficas Muriel. España. Recuperado de http://www.frasecelebre.net/Frases_De_Educacion.html

España, A. (2012). Los ateneos didácticos como dispositivos de formación y socialización de las prácticas en Sanjurjo, L. (2012), Los dispositivos para la formación en las prácticas profesionales. Ediciones Homosapiens. Santa Fe, Argentina.

Fallas, H y Ross, A (20014). Deserción estudiantil. Recuperado de http://www.nacion.com/gnfactory/especiales/2014/desercionestudiantil/index.html?sk=sanluisgonzaga

Guerrero, E. (2013). Deserción estudiantil: el punto negro tras las vacaciones de medio periodo. Recuperado de http://www.crhoy.com/ desercion-estudiantil-el-punto-negro-tras-las-vacaciones-de-medio-periodo/

Pérez, A. I. (1997). Socialización profesional del futuro docente en la cultura de la institución escolar: el mito de las prácticas. Revista Interuniversitaria de Formación del Profesorado, 29, 125140. Recuperado de http://dialnet.unirioja.es/descarga/articulo/117950.pdf

Programa Estado de la Nación en Desarrollo Humano Sostenible. (2013). Informe Estado de la Educación. Cuarto informe. Recuperado de: http://www.estadonacion.or.cr/files/biblioteca_virtual/educacion/004/5-Cap-1.pdf. 
Martínez, H. (2011). Docentes del siglo XXI: Innovar o innovar; en Soubiron, E; Rodríguez, D; Sanz, V; Conde, A. (coords.) (2011). La práctica pedagógica en entornos innovadores de aprendizaje. Seminario Internacional. Montevideo, Uruguay.

Sepúlveda, M.P. (2005). Las prácticas de enseñanza en el proceso de construcción del conocimiento profesional. Departamento de Didáctica y Organización Escolar Universidad de Málaga. 